\title{
Research on Self-healing Composite Materials
}

\author{
Peng Li \\ School of Mechanical and Electronical Engineering \\ East China Jiaotong University \\ Nanchang, PR China \\ ecjtulipeng@126.com
}

Tian Zou

School of Mechanical and Electronical Engineering East China Jiaotong University

Nanchang, PR China

861171294@qq.com

\begin{abstract}
In the using of materials, kinds of injury which is sometimes difficult to repair or too costly to repair may appear in the surface and interior, which will shorten the life of the materials. Self-healing technology can be used to improve material reliability and extend the service life, so composite materials with self-healing capability become an important research content of smart materials. Selfhealing materials can perform self-diagnosis after suffered from external forces and repair the crack or damage in a certain degree. According to the healing agents applied or not in the self-healing process, self-healing methods in polymer materials can be classified into extrinsic selfhealing and intrinsic self-healing. Based on the latest research achievements, several typical extrinsic selfhealing methods are summarized, including microcapsule self-healing, hollow-fiber self-healing and microcapsule self-healing. Several repairing mechanisms, self-healing system characteristic, existing problems and present research status are elaborated. Future research on selfhealing materials is prospected, optimizing and developing new healing agents system in order to improve the selfhealing efficiency and realizing the true smart materials.
\end{abstract}

Keywords- smart materials; composite materials; repairing mechanism; intrinsic self-healing; external selfhealing

\section{INTRODUCTION}

With the widely application of composite materials, their service life and safety become a hot topic at home and abroad. Materials inevitably produce cracks or other damages when suffered from external heat or force factors. This will result in the performance degradation of composite material, such as flexure strength, tensile strength, compression strength. Traditional restoration techniques, such as resin injection, mechanical connection subsides, and transfer subsidies, can repair material surface cracks. But for microscopic damage resulted in material internal microcracks can't apply above traditional repair techniques.

Based on hard reparative for internal microcracks, the US military firstly proposed self-healing material in the 1980s. The study of this type of problem has long been an important issue for both researchers and engineers and is part of a large futuristic concept known

\author{
Yuan Liu \\ School of Mechanical and Electronical Engineering \\ East China Jiaotong University \\ Nanchang, PR China \\ 1558669982@qq.com \\ Yuhua Wang \\ School of Mechanical and Electronical Engineering \\ East China Jiaotong University \\ Nanchang, PR China \\ 751494831@qq.com
}

as "smart structures" $[1,2]$. When the material produces microcracks and defects, itself has the ability to selfrestore in the absence of external effect, making composite materials for internal or external damage selfhealing, thereby eliminating the risk, enhancing material strength and prolong life.

\section{SELF-HEALING MECHANISM OF COMPOSITES}

The mechanism of composites self-healing derived from the principle of biological system's recover automatic. Many living biological system react to external damage by providing an autonomic healing response at the site of injury. For example, the skeletal bone enables biological fluids containing clotting agents, nutrients, and stem cells to flow from the network of blood vessels into a fractured region upon injury, facilitating over time the development of fibrocartillage, which calcifies into fibrous bone, and then dense lamellarbone. This process forms new bone that is essentially indistinguishable from uninjured bone tissue. Bone is a typical example of biological self-healing capabilities [3, 4].

Repair mechanism of self-healing materials can be broadly divided into intrinsic and external self-healing type. Intrinsic self-healing methods are mainly to provide energy for system through the heating system and other ways, making it crystallization, in the surface forming film or cross-linking to achieve self-healing [5], such as heat welding, thermo-reversible cross-linking reaction. External self-healing methods mainly depend on some kinds of functional substances dispersed or implanted in the materials. This paper has a brief introduction to relevant methods of the two self-healing mechanism in next sections.

\section{INTRINSIC SELF-HEALING}

\section{A. In situ thermal welding}

Thermal welding repair is based on non-covalent intermolecular. The hot plate welding is used to repair the internalcrack of thermoplastic polymers such as methyl methacrylate. Wetting and spread are two important parameters in the repair process. Wool $[6,7]$ studies the self-healing of the linear polystyrene and 
cross-linked vinyl resin composites and finds that the cracks have recovery of $1.7 \%$ after interface annealing, which is caused by linear polystyrene chains throughout.

\section{B. Thermo-reversible cross-linking reaction.}

In recent years, a kind of transparent polymeric material with highly cross-linked self-healing ability is presented. The crack of this material can be repaired repeatedlythrough the covalent reaction which needs a simple heat treatment. And the self-healing processing does not involve to additional catalyst or monomer molecules. Chen [8] introduces diels-alder (DA) thermal reversible copolymerization to funan and maleimide polymers, and proposes an iota submeshes linked reversible cross-linking covalently.This material has equivalent mechanical properties to the general resin such as epoxy and unsaturated polyester. The method provides a new way for exploring self-healing material.

\section{EXTERNAL SELF-HEALING}

From early literatures, most of published works in the self-healing field mainly focused on the external selfhealing mechanism. Namely, microencapsulation, hollow fiber or microvascular network embedded the repair agent is distributed in the composites. The repair agent is released when composite materials produce micro-cracks under external force; the crack voids combine to achieve self-healing.

\section{A. Microencapsulation}

As shown in Fig .1 (a), firstly the repair agent is filled in hollow capsules dispersed into the material. Secondly, in Fig.1 (b), when the materials subjected to shock or vibration, micro-cracks will appear and make hollow microcapsules rupture and healing agent outflow. Finally, in Fig.1 (c), the healing agent cures and makes crack healing under the conditions of catalyst.

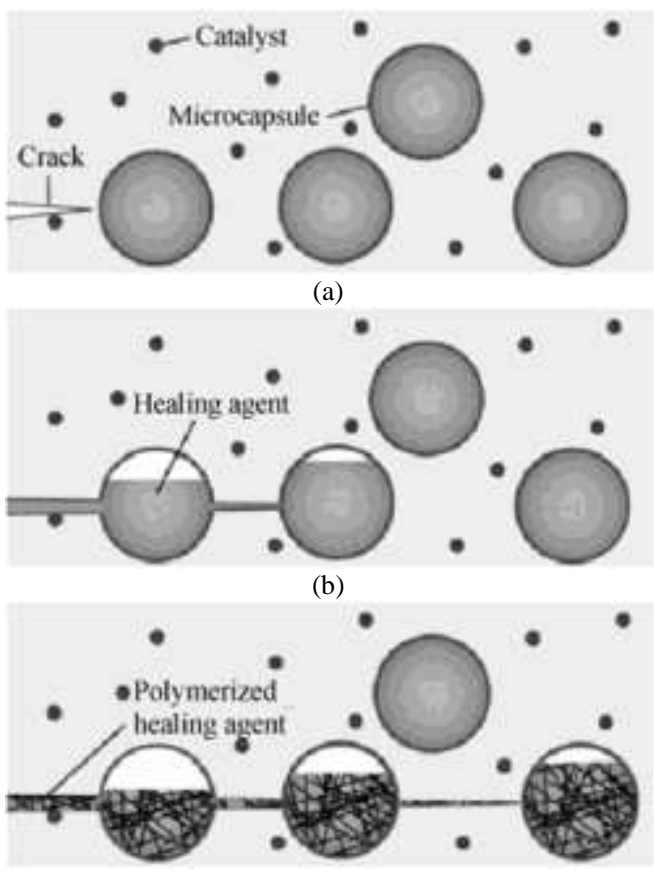

(c)

Figure 1. Principle of hollow microcapsules self-healing.
According to the above repair principle, White ${ }^{[1]}$ makes the dimer of cyclopentadiene (DCPD) entrapped in microcapsules of urea-formaldehyde resin, dispersed in low polymer of diethylenetriamine amine-cured epoxy resin together with the Grubbls catalyst. When microcapsules rupture, the DCPD rapidly penetratesinto cracks due to capillary siphon action, and exposure to Grubbls catalyst, then occurring activity ring-opening polymerization (ROMP) and quickly generate highly cross-linked polymeric network, finally achieving the purpose of repair. Experimental tests show that this material is able to recover $75 \%$ of the toughness. The repair system must meet the following three conditions for the microcapsule requirements: (1) the thick of capsule walls to moderate, not only to withstand the pressure of composite molding process, but also to feel the force brought by crack extension; (2) the hardness of the microcapsule not too strong; (3)the number and volume of implanted capsules to appropriate.

\section{B. Hollow fiber}

The self-healing principle is essentially same with microencapsulation, shown in Fig .2. The different just is the carrier of storing healing agent. When the material is subjected to impact and appear microcracks, the ruptured fibers release healing agent for curing materials.

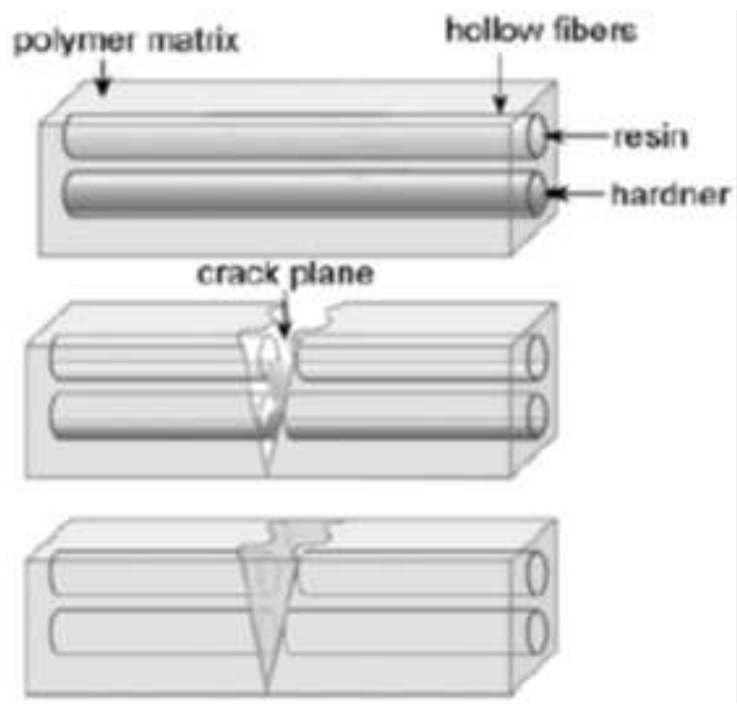

Figure 2. Principle of hollow fiber self-healing.

Cho $\mathrm{S}$ et al. embed tubes infused with resin and subjected the composite panels to low velocity impact [9]. The investigation, while demonstrating tube breakage and liquid healing agent release, is limited by the large diameter $(\mathrm{mm})$ capillaries used and the choice of liquid healing agent. Zhou Benlian et al [10] use epoxy resin and low molecular weight polyamide in proportion of 1:0.6 dubbing matrix. The repair fiber, filled with white latex, is molded layer by layer. And then the matrix is made three-point bending test, loading force until the crack appears. The study finds that when the slender glass tubes rupture, the inner repair agent automatically outflowing, the matrix cracks are bonded after a period of time. The experiment has measured that the average strength of epoxy after healing is $84 \%$ of the 
original strength. Bond et al. have developed a process to optimize the production of hollow glass fiber [11, 12] and have used those fibers (filled with liquid healing agents) in PMC for both damage detection and selfhealing [13-15].

\section{Microvascular network}

An alternative approach to the hollow fiber is to design anovelinter connected microvascular network. This is the most biomimetic approach since it replicates the vascular system of many plants and animals, but it is difficult to achieve practically and at large scales in synthetic materials. However, only this type of system is compatible with incorporation of a circulatory system that continuously transports the necessary chemicals and building blocks of healing to the site of damage.

Toohey et al. [16, 17] use the direct ink writing (DIW) to produce a system that mimics the structure of skin, with an epoxy substrate containing a 3D vascular grid of micro-channels filled with monomeric fluid dicyclopentadiene (DCPD), and a surface coating with incorporated Grubbs' catalyst (Fig .3(a)). The fracture of the coating under four-point bending released the DCPD from the vertical channels into the crack plane, leading to contact with the catalyst and subsequent polymerization. Tests of the coatings after damage and healing showed partial recovery of fracture toughness. Most notably, due to the interconnected nature of the network, the monomer reservoir could be replenished, enabling up to seven consecutive healing events in a single sample. In an effort to extend the number of healing cycles, the researchers employed the DIW method to make single grids, and then utilized photopolymerization to isolate separate networks for two-part epoxy healing schemes.

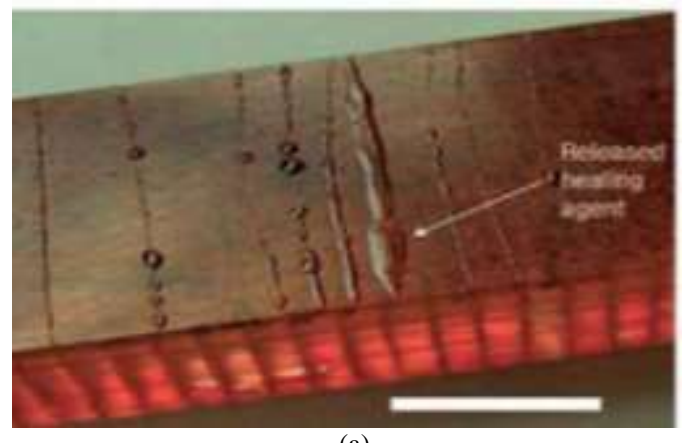

(a)

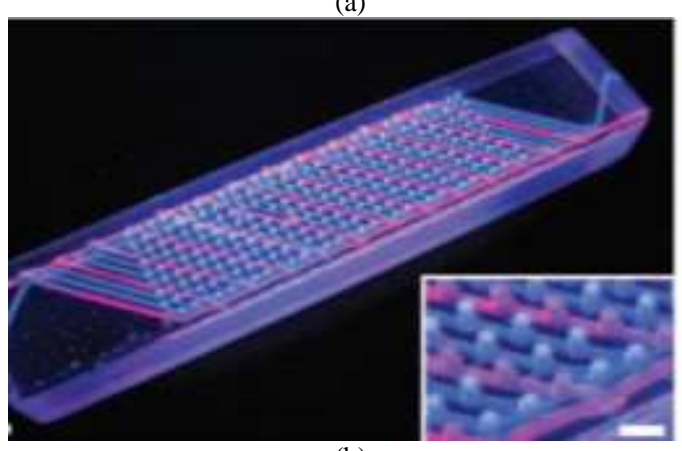

(b)

Figure 3.The example of direct-write microvascular network.

Hansen et al. [18] achieve greater control over network architecture through the use ofa ink writing method. The authors use microcrystalline wax ink that melts at high temperatures, as well as a block-copolymer ink that melts below room temperature. This advancement allows the writing of complex, separate, and interpenetrating networks toaid the mixing of healing components (Figure 3(b)). By creating vertical channels fromeach network in a number ratio, the number of healing cycles is further extended beyond 30 .

Kozola et al. [19, 20] have also used DIW to make networks for active-cooling by creating thin fin polymer specimens with embedded channels with varying network geometries. Using infrared imaging, the researchers examine the effects of flow rate, channel diameter,dimensionality, and cooling fluid on the transition from the heated specimen in theabsence of flow to the steady-state regime with flow as well as the effectiveness of steady-statecooling. Additionally, they apply micro-scale particle image velocimetry to measure flow rates in individual channels, in order to determine critical threshold flow rates foreffective channel cooling.

\section{SUMMARY}

Self-healing technology has profound significance to improve safety and reliability of product and great prospects for development and applications in a number of important engineering and cutting-edge technology. The biggest difficulty of intelligent self-healing technology lies in how to detect the location and extent of fracture damage inside the material and how to suppress or repair damage and fracture, which would involve sensing technology, microelectronics, materials science and artificial intelligence and structural engineering and other disciplines, along with the cross and the development of the discipline, to solve the problem which are not too difficult. Although the application and research of intelligent self-repairing material are still in its infancy, to be a new breakthrough in many ways, it is still a bright future and will become a research focus of materials science and engineering fields.

\section{ACKNOWLEDGMENT}

The authors gratefully acknowledge the financial support provided by the National Natural Science Foundation of China (Grant No. 51365012).

\section{REFERENCES}

[1] S.R. White, N.R. Sottos and P.H. Geubelle, "Autonomic Healing of Polymer Composites," Nature, vol. 415, 2002, pp. 817.

[2] E.B. Murphy and F. Wudl. "The World of Smart Healable Materials." Progress in Polymer Science, 35, 2010, pp. 223-251.

[3] N.E. Brown and N.R. Sottos a, "Fracture Testing of a Selfhealing Polymer Composite," Experimental Mechanics, vol. 42, 2002, pp. 372-379.

[4] M.W. Keller and N.R. Sottos, "An Elastomeric Self-healing Material." In proceedings of the 2006 SEM Annual Conference and Exposition on Experimental and Applied Mechanics, vol. 1, 2006, pp. 379-382.

[5] B.J. Blaiszik, S.L. Kramer and S.C. Olugebefola, "Self-healing Polymers and Composites." Annual Review of Materials Research, vol. 40, 2010, pp. 179-211.

[6] P.R. Wool and P. Richard. "Self-healing Materials: a Review." Soft Matter. vol. 4. 2008. pp. 400-418. 
[7] P.R. Wool, A. Campanella and M.L. Bonnaillie, "Polyurethane Foams from Soyoil-based Polyols," Journal of Applied Physics, vol. 112, 2009, pp. 2567-2578.

[8] X.X. Chen, A.M. Dam and A. Kono, "Thermally Remendable Cross-linked Polymeric Material," Science. vol. 295, 2002, pp. 1698-1702.

[9] S. Cho and H. Andersson, "Polydimethylsiloxane-Based SelfHealing Materials," Advanced Materials, vol. 18, 2006, pp. 9971000.

[10] B.L. Zhou, R.C. and Wang, Y. Zeng, "The Bionic Study of Water Flow Adaptive Control Intelligent Gel," Journal of Materials Research, vol. 1, 2005, pp. 42-46.

[11] M. Hucker, A. Foreman and J. Hudd. "Optimisation of Hollow Glass Fibers and Their Composites." Advanced Composites, vol. 8, 2006, pp. 181-189.

[12] R.S. Trask and G. J. Williams, "Bioinspired Self-healing of Advanced Composite Structures Using Hollow Glass Fibers." Journal of the royal society Interface, vol. 4, 2007,pp. 363-371.

[13] J.W.C. Pang and I.P. Bond, "Bleeding Composites Damage Detection and Self-repair Using a Biomimetic Approach." Composites Part A, Applied Science and Manufacturing, vol. 36, 2005, pp. 183-188.

[14] G. Williams and R. Trask, "A Self-healing Carbon Fiber Reinforced Polymer for Aerospace Applications." Applied Science and Manufacturing, vol. 38, 2007, pp. 1525-1532.
[15] J.W.C. Pang, and I.P. Bond, "A Hollow Fiber Reinforced Polymer Composite Encompassing Self-healing and Enhanced Damage Visibility." Composites Science Technology, vol. 65, 2005, pp. 1791-1799.

[16] K.S. Toohey, N.R. Sottos, J.A. Lewis and J.S. Moore, "Selfhealing Materials with Microvascular Networks," Nature Materials, vol. 6, 2007, pp. 581-585.

[17] K.S. Toohey, C.J. Hansen, J. Lewis and N.R. Sottos, "Delivery of Two-part Self-healing Chemistry via Microvascular Networks," Advanced Function Materials, vol. 19, 2009, pp. 1399-1405.

[18] C.J. Hansen, K.S. Toohey, N.R. Sottos and J.A. Lewis, "Selfhealing Materials with Interpenetrating Microvascular Networks," Advanced Materials, vol. 21, 2009, pp. 4143-4147.

[19] B.D. Kozola, V.K. Natrajan and K.T. Christensen, "Active Cooling of Polymer Structures Using Microvascular Networks," In: 45th SES Technical Meeting, University of Illinois at Urbana-Champaign, Urbana, IL. 2008

[20] B.D. Kozola and L.A. Shipton "Characterization of Active Cooling and Flow Distribution in Microvascular Polymers,' Journal of Intelligent Material Systems and Structures, 2010. 